\title{
Erratum to: Contribution of two molecular assays as compared to selective culture for MRSA screening in a low MRSA prevalence population
}

\author{
E. Nulens $\cdot$ P. Descheemaeker $\cdot$ R. H. Deurenberg $\cdot$ \\ E. E. Stobberingh $\cdot$ B. Gordts
}

Published online: 3 July 2010

(C) Urban \& Vogel 2010

Erratum to: Infection (2010) 38:98-101

DOI 10.1007/s15010-009-9117-0

There was an error in the "Results" section of the abstract.

The corrected paragraph reads as follows:

Results: Eight MRSA carriers were detected by selective culture (1.6\% prevalence). The sensitivity, specificity, positive predictive value, and negative predictive value were $62.5,99.0,50.0$, and $99.4 \%$ for BD GeneOhm ${ }^{\mathrm{TM}}$ StaphSR and 62.5, 97.7, 31.3, and 99.4\% for Cepheid Xpert $^{\mathrm{TM}}$ MRSA, respectively.

The online version of the original article can be found under doi:10.1007/s15010-009-9117-0.

E. Nulens $(\bowtie) \cdot$ P. Descheemaeker · B. Gordts Medical Microbiology, General Hospital Sint-Jan AV Brugge-Oostende, Ruddershove 10, 8000 Brugge, Belgium e-mail: eric.nulens@azbrugge.be

E. Nulens · R. H. Deurenberg · E. E. Stobberingh Medical Microbiology, Maastricht University Medical Center, P.O. Box 5800, 6202 AZ Maastricht, The Netherlands 